\title{
Predicting population exclusion for services dependent on mobile digital interfaces
}

\author{
Michael Bradley* \\ University of Cambridge \\ mdb54@cam.ac.uk
}

Jakob Kluge

Institut für Zukunftsstudien und Technologiebewertung j.kluge@izt.de

\author{
Joy Goodman-Deane \\ University of Cambridge \\ jag76@cam.ac.uk \\ P. John Clarkson \\ University of Cambridge \\ pjc10@cam.ac.uk
}

\begin{abstract}
Mobile services accessible solely via digital interfaces are becoming increasingly common. This may result in the exclusion of many potential users because they lack the access to the required digital technologies, the capability to operate the interfaces or the attitudes for successful technology engagement. This paper focuses on the issue of access as a first step in understanding this exclusion. Analysis from a recent population-representative survey in Germany $(n=1010)$ provides a baseline exclusion prediction for mobile services with different technology requirements, for example a service that requires the user to install an app on a smartphone versus or a service that provides information via a website on a smartphone. The analysis has been carried out on the whole population and on selected groups that are potentially at greater risk of being excluded from these services, such as those over the age of 65 , women and those with low educational achievement. The results suggest that services that only offer access via an app, which needs installation on a mobile device, can exclude significant numbers of potential users.
\end{abstract}

\section{CCS CONCEPTS}

- Human-centered computing $\rightarrow$ Human computer interaction (HCI); HCI design and evaluation methods; User models; Human computer interaction (HCI); HCI design and evaluation methods; User studies; Human computer interaction (HCI); HCI design and evaluation methods; Heuristic evaluations; Accessibility; Empirical studies in accessibility; Accessibility; Accessibility design and evaluation methods.

\section{KEYWORDS}

Inclusive Design, Digital Mobility Services, Digital exclusion

\section{ACM Reference Format:}

Michael Bradley, Joy Goodman-Deane, Jakob Kluge, and P. John Clarkson. 2021. Predicting population exclusion for services dependent on mobile

${ }^{*}$ Lead author for contact.

\section{(c) (i) (8)}

This work is licensed under a Creative Commons Attribution-NonCommercial International 4.0 License.

MobileHCI '21 Adjunct, September 27-October 01, 2021, Toulouse \& Virtual, France (c) 2021 Copyright held by the owner/author(s).

ACM ISBN 978-1-4503-8329-5/21/09.

https://doi.org/10.1145/3447527.3474862 digital interfaces. In Adjunct Publication of the 23rd International Conference on Mobile Human-Computer Interaction (MobileHCI '21 Adjunct), September 27-October 01, 2021, Toulouse \& Virtual, France. ACM, New York, NY, USA, 5 pages. https://doi.org/10.1145/3447527.3474862

\section{INTRODUCTION}

Digital and computing technology has facilitated significant benefits to services for individuals and for society for many years now. Relatively recently, the ability for individuals to directly access the services via digital interfaces has been facilitated by the internet, and more recently by smartphone technology and rapid data connectivity on-the-go via mobile cellular networks.

Originally individual consumer access to digitally facilitated services was predominantly via intermediary customer service agents, who would act as a conduit between the consumer and the digital technology, which they were trained to operate. Examples of this model include travel agencies, banks, local government service providers and conventional 'bricks and mortar' shops [1].

Since the internet has enabled individuals to directly access services through digital interfaces, many of the service providers have reduced or eliminated the human customer service mediators. The benefits of supplying the services directly to the consumer have included reduced costs for the service provider [2].

Some of the benefits of this direct interaction with the digital services are passed back to the consumer, usually in the form of reduced costs, efficiency of transaction and convenience. These benefits can also be considerable for some groups that are at potentially greater risk of being excluded from these services (called vulnerable-to-exclusion groups in this paper). These include those with disabilities, where the service can be specially adapted or designed to be intrinsically accessible.

However, digital interface mediated services are only useful for those who can access and use them effectively. People may be effectively excluded due to the need for users to have the necessary hardware and connectivity, sufficient digital competence (or experience) or the motivation and willingness to learn and explore an interface $[3,4]$.

Therefore, it is very useful for service providers to be able to understand both the numbers, and who in the population, would be excluded from using a service delivered by digital mediation so that decisions about how to accommodate those who might otherwise be excluded can be made, and to inform the presentation and design of the service $[3,5]$. Some of this population data is available for many regions through government sponsored national surveys of 
internet and mobile phone use, and from research agencies such as Pew Research, e.g. [6]. However, the combination of information required to develop exclusion estimates, such as individual participant access and frequency of use of a smartphone, on the go internet use and frequency of app installation was not available.

This paper uses population representative survey data, described in [4] to examine the numbers excluded from different types of mobile services on the grounds of technology access requirements. This is the first step in a more complete exclusion analysis that could also look at digital competence, experience and attitudes. We examine how the exclusion varies for different types of mobile services for the population in Germany as a whole, and for different vulnerable-to-exclusion groups.

\subsection{Overview of the wider DIGNITY project}

The survey described in this paper was conducted as part of the DIGital traNsport In and for socieTY (DIGNITY) research project. This project is investigating how to foster a sustainable, integrated and user-friendly digital travel eco-system that improves accessibility and social inclusion [7]. It particularly focuses on reducing exclusion for groups that have been identified as being particularly vulnerable to digital mobility exclusion. Although the focus of this project is on digital mobility services, the findings presented in this paper are of relevance to a wide range of mobile services, including tourism, information systems and many others, as well as transport.

\subsection{Background: Digital exclusion and digital interface exclusion}

1.2.1 Digital exclusion and digital interface exclusion. 'Digital exclusion' has generally been used to describe the condition of not having access to the internet $[8,9]$. The reasons why an individual might experience digital exclusion include poverty, lack of digital skills, lack of suitable devices and concerns about security. In addition, some people choose not to engage with the internet for reasons such as a lack of interest or perceived need.

'Digital interface exclusion' is proposed by the authors as a term to describe the condition of not being able to operate a digital interface due to a lack of skill, confidence or willingness to engage and explore an interface to be able to achieve a goal. Typically those who experience digital interface exclusion will also not engage with the internet, and so would be considered digitally excluded.

1.2.2 Factors affecting digital interface exclusion. Digital interface exclusion arises due to a mismatch between the interface's attributes and the user's characteristics, within a socio-technical context of use [3]. These user characteristics extend beyond basic user capabilities to include technology access, technology experience, intentions, attitudes, learning and competence on component tasks [3, 10]. This is somewhat related to the work on Technology Acceptance Models, e.g. [11], originally proposed by [12], which identify factors affecting an individual's technology adoption journey. However, digital interface exclusion differs from technology adoption as it focuses on the effective use of interfaces in practice, and therefore more accurately reflects the nature of digital interactions in day-today activities.
1.2.3 Calculating estimates of population exclusion. Often when designing products and services, trade-offs have to be made between cost and quality. When attempting to design inclusive products and services, a key quality question has to be 'how many people will we exclude if we design it this way, versus that way?'. The University of Cambridge's exclusion calculator [13] has been developed to attempt to provide estimates from a human capability perspective, which answer that question. Its power lies not only in the ability to provide a more objective rationale for decision making in the service design and development process, but also with potential to help justify decisions that otherwise might appear of poor value.

\section{METHOD}

\subsection{Categorisation of technology requirements}

Digital mobile services include any digital technology mediated service as well as those which also offer alternative (non-digital) means of access. They include car sharing platforms, ticketing machines, online journey planning tools, and ride hailing applications. As a part of the DIGNITY project, a 'Digital Mobility Services Good Practice' examples list was created to provide service specifier, providers and developers with guidance on how to improve inclusivity of service. This challenged the team to find a way to provide high-level population exclusion estimation figures, when they were unable to access all of the services and carry out a detailed digital interface exclusion audit on each.

It was recognised that a high proportion of the overall digital interface exclusion could be predicted based simply on the type of digital service, given the percentages of the populations that do not have access to certain digital technologies, nor potential access to the interfaces of the digital mobility services. This would provide a 'baseline' level for the overall digital interface exclusion for a service, which would also need to account for the difficulty and complexity of a particular interface and service design.

Categories of service interfaces were developed which could be analysed to give the baseline exclusion estimation. These were chosen to reflect the relevant data from the survey, and the key characteristics that may affect the digital access exclusion. They range from fixed installation devices (such as ticket machines), which have no user managed hardware to cause exclusion, through a basic mobile phone interface (for calling or texting to interact), to apps requiring installation on a smartphone and mobile internet connectivity, such as Waze for route guidance in online mode. The complete list of these appears in Table 2.

In Table 1 the relationship between the characteristics of a particular digital mobility system are mapped to the appropriate variables from the survey. This takes into account the direct access to the digital service interface (e.g. for a ticket machine), but also the implied task step required to get access to a particular interface, which is relevant for apps. Most services which require an app interface, unless the app is preinstalled on the operating system, intrinsically require the app to be found, downloaded and installed by the end user. Not taken into consideration at this 'baseline' level calculation is the need in some cases for a complex registration process to be completed before service access is available.

\subsection{Survey}


Table 1: Survey variables corresponding to each digital technology requirement.

\begin{tabular}{ll}
\hline Digital technology requirement & Variables from survey \\
\hline Smartphone access & Ownership of a smartphone \\
Access to any computing device & Access to a computer OR tablet OR ownership of a smartphone \\
Mobile internet access & Internet access in any location AND has used internet on a \\
& smartphone in the last 3 months \\
Internet access in general & Internet access in any location \\
Ability to send and receive calls and texts while on the move & Own any mobile phone \\
Ability to install apps & Installed an app on a smartphone OR tablet in the last 12 months, \\
& AND has internet access. \\
\hline
\end{tabular}

2.2.1 Overview of survey. A survey was designed to examine the prevalence of digital behaviours, attitudes and competence amongst regional and national populations. The survey is being conducted in five different European countries (Belgium, Germany, Italy, the Netherlands and Spain). The surveys in some of these countries are still underway, having been delayed due to COVID-19 restrictions. This paper uses initial results from the first completed survey, which was in Germany. Initial results and a fuller description of this survey are in [4].

The German survey was conducted by forsa, an independent market and opinion research institute. Participants completed the questionnaire with an interviewer. Each interview took 20 to 30 minutes. Approval was obtained from the University of Cambridge Engineering Department ethics committee.

2.2.2 German sample. A three-stage face-to-face sampling design called ADM was deployed to obtain a population-representative sample of 1010 respondents aged 16 and above. The ADM sampling framework was utilized as it is widely used in market, media and social research in Germany. After sample locations are chosen, private households and target persons within these households are selected at random using a random-route procedure. At least four contact attempts are made for each target household or person, and incentives are not offered to participants. The ADM framework is maintained by an association representing Germany's 70 largest commercial market and social research organizations and includes a code of conduct that sets out ethical guidelines based on the ICC/ESOMAR International Code on Market, Opinion and Social Research and Data Analytics.

The resultant sample was $48.4 \%$ male and $51.6 \%$ female. The age bands were: $33.3 \%$ aged $16-39,41.2 \%$ aged $40-64,12.0 \%$ aged 65-74 and $13.5 \%$ aged 75 and over. $81.7 \%$ used a smartphone at least once a week. The sample was weighted to more closely match the population on region, age and gender. All the results in this paper use this weighting.

2.2.3 Questionnaire. The survey questionnaire was adapted from a previous UK survey conducted in 2019 [3]. The questionnaire was developed in English and then translated into German and into the other survey languages by professional translators. They were subsequently re-translated back into English and checked by the survey creators before improvements were made and the translations finalised.
The questionnaire contained sections covering: technology access and use, use of technology for transport, attitudes towards technology, basic digital interface competence, sensory, motor and cognitive capabilities, and demographics. This paper focuses on some of the questions in the sections on technology access and use from the German survey.

Within these sections, participants were asked multiple-choice self-report questions regarding their access to, and frequency of use, of the internet, computers, tablets and smartphones. They were subsequently asked whether they had performed various technologyrelated activities recently, such as whether they had installed an app on a smartphone or tablet within the last 12 months. These were based on questions from the Internet Access Survey 2017 [14] to allow for comparison with national UK statistics.

\subsection{Exclusion analysis method}

The analysis was conducted in SPSS v27 with the dataset weighted by region, age and gender to better match the German population as a whole.

2.3.1 Variables used for each of the technology requirements. Variables from the survey were identified for each of the technology requirements associated with digital mobile services (see Table 1). In some cases, there was no survey variable that was an exact match for a requirement. In these cases, variables were chosen to approximate the requirement. Participants giving a negative response to the variable would be likely to be excluded from the technology requirement.

In particular, the survey did not ask if participants had mobile internet access. Instead, it asked whether the participant had used internet on a smartphone in the last 3 months. Although this internet use was not necessarily mobile (as it could be through home Wi-Fi only), it provides a baseline. Someone who hadn't used the internet on a smartphone in the last 3 months would be unlikely to have mobile internet access.

For installation requirements, we examined whether the participant had installed an app on a smartphone or tablet in the last 12 months. Although people who have not installed apps may still be capable of doing so, they are likely to experience difficulty with this task and be reluctant to do so.

2.3.2 Specific groups examined. The exclusion figures were examined for some subgroups to highlight how exclusion might vary depending on the intended user population. These groups were 
Table 2: Exclusion based on technology access requirements only for whole population and selected groups that are potentially more vulnerable to exclusion. Note that the exclusion may be higher in practice, when the difficulty of use of the digital interface is also considered.

Exclusion (\% of group who do not meet the basic technology access requirements to use this type of service)

\begin{tabular}{|c|c|c|c|c|c|}
\hline Type of service interaction & Whole population & Women & Low income & $\begin{array}{l}\text { Low } \\
\text { education }\end{array}$ & Older people \\
\hline $\begin{array}{l}\text { A fixed installation (e.g. screen, kiosk, machine) } \\
\text { in a public location that does not require any } \\
\text { other equipment. }\end{array}$ & $0 \%$ * & $0 \%$ * & $0 \%$ * & $0 \%$ * & $0 \%$ * \\
\hline $\begin{array}{l}\text { A service that requires the user to have a mobile } \\
\text { phone (e.g. to receive a text or make a call) }\end{array}$ & $2.1 \%$ & $3.2 \%$ & $5.5 \%$ & $4.4 \%$ & $7.9 \%$ \\
\hline $\begin{array}{l}\text { Website accessed via any device in a location (e.g. } \\
\text { home) that does not require mobile internet } \\
\text { access. }\end{array}$ & $10.8 \%$ & $11.5 \%$ & $18.8 \%$ & $21.2 \%$ & $40.5 \%$ \\
\hline $\begin{array}{l}\text { Pre-installed smartphone app that does not } \\
\text { require mobile internet access }\end{array}$ & $17.7 \%$ & $17.0 \%$ & $28.1 \%$ & $32.9 \%$ & $55.8 \%$ \\
\hline $\begin{array}{l}\text { Pre-installed smartphone app or a website that } \\
\text { requires mobile internet access }\end{array}$ & $19.2 \%$ & $18.0 \%$ & $30.6 \%$ & $34.6 \%$ & $59.6 \%$ \\
\hline $\begin{array}{l}\text { Smartphone app that requires installation but } \\
\text { not mobile internet access }\end{array}$ & $47.3 \%$ & $52.3 \%$ & $64.2 \%$ & $71.5 \%$ & $85.5 \%$ \\
\hline $\begin{array}{l}\text { Smartphone app that requires installation and } \\
\text { mobile internet access }\end{array}$ & $47.4 \%$ & $52.4 \%$ & $64.2 \%$ & $71.5 \%$ & $85.5 \%$ \\
\hline
\end{tabular}

* The figures in this table, including the $0 \%$ refer to the exclusion caused solely by the constraints of access to the interface and do not include the exclusion caused by the difficulty of the interface itself. The overall digital interface exclusion for a fixed installation will be greater than $0 \%$, as there is likely to be significant additional exclusion associated with the interface itself depending on its design.

selected because they are at potentially greater risk of exclusion from mobile digital services. The analysis in this paper adds to the understanding of the distribution of these groups and the proportions within them that are likely to experience exclusion from mobile digital services in practice. It is important to note that the groups are neither independent nor homogeneous. All of the groups contain people with high as well as low levels of technology access and competence. Note also that these groups are not independent:

- Older people (25.3\% of sample): those aged 65 and over.

- Women (51.0\%): those giving their gender as female.

- Low education (32.2\%): those listing their highest level of education as secondary general school-leaving certificate or below. This roughly corresponds to ISCED levels 0-2 [15]. Those currently attending general school are not included in this group.

- Low income (14.1\%): those with a net monthly household income below a poverty line of 1040 euros for a single-person household [16]. The poverty line for multi-person households was calculated from this based on the OECD-modified household size [17].

\section{RESULTS AND DISCUSSION}

Table 2 shows the results of the calculations based on the whole population sample, and for specific vulnerable-to-exclusion groups for different types of service interactions. It is worth noting that these figures represent calculated estimates of baseline exclusions, solely attempting to quantify the technology access requirements for potential users to be able to interact with the service interface. Many more people could be excluded based on digital competence and attitudes, depending on how the interface itself is designed. In particular, fixed digital installations have a baseline technology access exclusion of $0 \%$, as the interface technology is supplied by the service provider. However, if the exclusion resulting from the complexity of interaction is added, then some of them are likely to exhibit high exclusion because the interfaces are impossible to use for some potential users.

The results show that significant portions of the population do not have access to the services and devices necessary to use digital mobility offerings, and that these access barriers run along socioeconomic dimensions. This is consistent with current research about digital exclusion and internet non-use as well as research concerning disparities in access to the smart mobility ecosystem [18-20]. Taking a closer look at specific numbers, some similarities can be found as well when compared with recent surveys that attempt to map the "digital divide" in Germany: For example, representative studies indicate that for people over 50 years in Germany, $36 \%$ of people do not own a smartphone and that significant differences also exist with regard to educational level and gender [21]. This also applies to the proportion of people who use the Internet. Among young people, this proportion is almost twice as high as among old people (98\% in the $14-19$ age group compared with $52 \%$ in the over-70 age group).

In general, exclusion across the whole population is lower than in the groups identified as being potentially vulnerable-to-exclusion, 
with the exception of women, where the figures are fairly similar to the whole population. Indeed, women have a slightly lower exclusion for some types of services. Note this analysis has been done for women as a group. Some subgroups may be more vulnerable to exclusion than others. Further analysis is needed to explore interactions between gender and other variables.

The low income and low education groups have higher exclusion than both the whole population group, and women group, but the highest levels of exclusion for all types of digital service is experienced by the older group.

The results for the services that require any type of mobile phone will only exclude about $2 \%$ of the German population from a technology access perspective alone, but this rises to nearly $8 \%$ for aged 65 or older. Those with low educational achievement have exclusion approximately double that of the general population, and for those with low income it is about $5 \%$. Since the survey did not ask where the mobile phone is kept, nor any aspects of digital interface exclusion, the actual exclusion may be significantly higher.

Website access in a fixed location precludes about $11 \%$ of the German population rising to about $40 \%$ of older people. Services that rely only on users having a smartphone but not needing mobile data nor to download an app have a baseline exclusion of c. $18 \%$ of the population, rising to $28 \%$ for the low income group and $33 \%$ for the low education group. For the older group the calculated exclusion is nearly $56 \%$. When the service requires mobile connectivity, this increases exclusion by a small amount for all groups.

For smartphone access requiring the downloading and installing an app, the predicted exclusion figures are dramatically increased due to the calculation assumption that those who have not downloaded an app in the previous 12 months are not going to want, or be able to do so for a particular service. Thus exclusion for app use not requiring mobile internet connectivity rises to about $47 \%$ of the whole population, rising to $85 \%$ for the 65 's and over. For apps that require mobile internet connectivity, this is predicted to rise by $0.1 \%$ only for the whole population group and women. Clearly, this represents an opportunity for service providers to support potential users in the downloading of apps with which to access their service.

\section{CONCLUSIONS AND FURTHER WORK}

Services mediated by digital interfaces alone are potentially highly exclusive for the general population and very highly exclusive for older people, those on low income and those with low educational achievement. Supporting potential users to download and install apps through analogue means, such as helpers and community ambassadors may reduce exclusion caused by app installation. Services that aspire to be as inclusive as possible need to offer non-digital mediation to reduce exclusion. This is the first step in a more complete exclusion analysis that would also look at digital competence, experience and attitudes. The survey described in this paper is currently underway or completed in four other European countries. An earlier version of the survey was also conducted in the UK. Further work will compare the findings between these countries to get a more complete picture for European populations.

\section{ACKNOWLEDGMENTS}

This research was done as part of the DIGNITY project which received funding from the European Union's Horizon 2020 research and innovation programme under grant agreement number 875542 . We would like to thank all the research partners on this project for their input into designing and carrying out the survey.

\section{REFERENCES}

[1] Age UK. 2018. Digital Inclusion Evidence Review. Retrieved 31 May 2021 from https://www.ageuk.org.uk/globalassets/age-uk/documents/reports-andpublications/age_uk_digital_inclusion_evidence_review_2018.pdf

[2] Deloitte.2013. The Digital Transformation of Digital Services. Retrieved 30 June 2021 from https:/www2.deloitte.com/content/dam/Deloitte/nl/Documents/ consumer-business/deloitte-nl-the-digital-transformation-of-customerservices.pdf

[3] Joy Goodman-Deane, Mike Bradley, Sam Waller, P. John Clarkson. 2020. Quantifying exclusion for digital products and interfaces. CWUAAT 2020 (10th Cambridge Workshop on Universal Access and Assistive Technology), Cambridge, UK, March 2020, Springer

[4] Joy Goodman-Deane, Jakob Kluge, Elisabet Roca Bosch, Nina Nesterova, Mike Bradley, P. John Clarkson. 2021. Digital mobility services: A population perspective. In: Proceedings of British HCI 2021. London, UK. 19-21 July 2021

[5] Sam Waller, Mike Bradley, Ian Hosking, P. John Clarkson. 2013. Making the case for inclusive design. Applied ergonomics. 46. 10.1016/j.apergo.2013.03.012.

[6] Andrew Perrin. 2021. Pew Research Center report: Mobile Technology and Home Broadband 2021, available at https://www.pewresearch.org/internet/wp-content/ uploads/sites/9/2021/06/PI_2021.06.03_Mobile-Broadband_FINAL.pdf

[7] Dignity project. 2021. Retrieved 31 May 2021 from https://www.dignity-project. $\mathrm{eu} /$.

[8] Government Digital Inclusion Strategy. 2014. Retrieved on 23 April 2021 from GOV.UK. https://www.gov.uk/government/publications/government-digitalinclusion-strategy/government-digital-inclusion-strategy

[9] Helen Milner.(Ed.). 2007. Digital Inclusion A discussion of the Evidence Base. UK Online \& Freshminds.

[10] Nicole M. Wagner, Khaled Hassanein, Milena Head. 2010. Computer use by older adults: A multi-disciplinary review. Computers in Human Behavior, 26, 870-882

[11] Yvonne Barnard, Mike Bradley, Francis Hodgson, Ashley Lloyd. 2013. Learning to use new technologies by older adults: Perceived difficulties, experimentation behaviour and usability. Computers in Human Behavior, 29, 1715-1724

[12] Fred D. Davis. 1989. Perceived Usefulness, Perceived Ease of Use, and User Acceptance of Information Technology. MIS Quarterly, 13(3), 319-340

[13] P. John Clarkson, Sam Waller, Carlos Cardoso. 2014. Approaches to estimating user exclusion. Applied Ergonomics. https://doi.org/10.1016/j.apergo.2013.03.001

[14] Office for National Statistics. 2017. Internet Access - households and individuals, Great Britain: 2017. Retrieved on 30 April 2021 from https://www.ons.gov.uk/peoplepopulationandcommunity/ householdcharacteristics/homeinternetandsocialmediausage/bulletins/ internetaccesshouseholdsandindividuals/2017

[15] Eurostat. Undated. International Standard Classification of Education (ISCED) Retrieved on 30 April 2021 from https://ec.europa.eu/eurostat/statisticsexplained/index.php/International_Standard_Classification_of_Education (ISCED)\#Implementation_of_ISCED_2011_.28levels_of_education.29

[16] Statistisches Bundesamt(German Federal Statistical Office). 2021. Risks of poverty have become further entrenched in Germany. https://www.destatis.de/EN/Press/ 2021/03/PE21_113_p001.html (retrieved Apr 2021).

[17] OECD (undated) Adjusting household incomes: equivalence scales. Retrieved on 30 April 2021 from https://www.oecd.org/economy/growth/OECD-NoteEquivalenceScales.pdf

[18] Aaron Golub, Vivian Satterfield, Michael Serritella, Jai Singh, \& Senna Phillips. 2019. Assessing the barriers to equity in smart mobility systems: A case study of Portland, Oregon. Case Studies on Transport Policy, 7(4), 689-697. https: //doi.org/10.1016/j.cstp.2019.10.002

[19] Soren Groth. 2019. Multimodal divide: Reproduction of transport poverty in smart mobility trends. Transportation Research Part A: Policy and Practice, 125, 56-71. https://doi.org/10.1016/j.tra.2019.04.018

[20] Ellen J. Helsper, Bianca C Reisdorf. 2017. The emergence of a "digital underclass" in Great Britain and Sweden: Changing reasons for digital exclusion. New Media \& Society, 19(8), 1253-1270. https://doi.org/10.1177/1461444816634676

[21] Pew Research Center. 2019. In emerging economies, smartphone adoption has grown more quickly among younger generations. Retrieved on 29 June 2021 from: https://www.pewresearch.org/global/2019/02/05/in-emergingeconomies-smartphone-adoption-has-grown-more-quickly-among-youngergenerations/ 